\title{
A universal relation for the stress dependence of activation energy for slip in body-centered cubic crystals
}

\author{
A. H. W. Ngan a) and H. F. Zhang ${ }^{\text {b) }}$ \\ Department of Mechanical Engineering, The University of Hong Kong, Pokfulam Road, \\ Hong Kong, People's Republic of China
}

(Received 6 January 1999; accepted for publication 19 April 1999)

\begin{abstract}
By analyzing experimental data in the literature, the activation energy $H$ for slip in body-centered cubic metals is found to approximately obey the simple relation $H \approx 0.1 \mu b^{3}(1-t)^{2}$, where $t$ is the applied stress normalized by the zero-temperature Peierls stress, $\mu$ is the $\langle 111\rangle$ shear modulus, and $b$ the $1 / 2\langle 111\rangle$ Burgers vector. Such universal relation is explained by analyzing the activation processes of kink-pair generation and expansion along threefold screw dislocations using the generalized Peierls-Nabarro model [A. H. W. Ngan, J. Mech. Phys. Solids 45, 903 (1997)]. The model also predicts qualitatively the general form of the orientation dependence of Peierls stress at zero temperature. (C) 1999 American Institute of Physics. [S0021-8979(99)03715-9]
\end{abstract}

\section{INTRODUCTION}

Most of the current understanding of the behavior of screw dislocation cores in the body-centered cubic (bcc) lattice is by and large derived from atomistic simulation studies performed in the last three decades or so (see, e.g., review by Duesbury $\left.{ }^{1}\right)$. These atomistic investigations lead to the current perception that (i) $1 / 2\langle 111\rangle$ screw dislocation cores are in general threefold dissociated with intrinsically high Peierls stress of the order of $10^{-2} \mu, \mu$ being the shear modulus, (ii) their slip behavior violates Schmid's law, (iii) their motion at zero temperature may follow a path along $\{101\}$ or a zig-zag path averaging along a twinning or antitwinning $\{112\}$ plane, and (iv) their motion can be affected by nonglide stress components. At finite temperatures, motion of screw dislocations does not happen in a plane-strain manner but is effected by the generation and nucleation of kink pairs. At low deformation temperatures, it is the nucleation of these kink pairs that forms the rate determining step for slip. The investigation of kink-pair generation using three-dimensional (3D) molecular dynamics (MD) is currently underway in a number of laboratories world wide, ${ }^{2}$ but until the emergence of their results, and probably even after that, most of the concepts regarding kink-pair activation are still based upon the continuum models. The continuum approaches clearly have their own drawbacks. In one group of theory due to Celli et al. ${ }^{3}$ and Dorn and Rajnak, ${ }^{4}$ the dislocation is treated as a piece of elastic string associated with a certain line tension moving under the influence of an assumed Peierls potential. The Peierls potential, being the energy potential of the dislocation with respect to its position, is purely phenomenological because the constraint that must be applied to keep the dislocation at a general nonequilibrium position cannot be clearly defined. Also, the introduction of an inner cutoff radius in the line-tension calculation disconnects the whole approach from the vast

\footnotetext{
a)Electronic mail: hwngan@hkucc.hku.hk

${ }^{b)}$ Permanent address: Central Iron and Steel Research Institute, Beijing 100081, P.R. China.
}

body of literature on the core behavior of the dislocation, and so the line-tension approach loses contact with many of the core-related characteristics of the bcc slip behavior such as the orientation dependence of Peierls stress. In another group of continuum theory due to Duesbury, ${ }^{5}$ the dissociation of the dislocation core into fractional dislocations is modeled by treating the fractional dislocations as Volterra dislocations. While the dissociation behavior under stress can be modeled and so the theory can claim to bear direct relation with core-related effects, the whole approach inherits all the limitations from the Volterra core, namely, that the cutoff radii are ill defined, and the stacking fault energies are unknown because in the bcc crystal structure, stacking faults are predicted to be unstable. ${ }^{6}$

In light of these difficulties, the present work aims at developing a semicontinuum model which combines certain elements of the atomistic and continuum picture of the dislocation kink-pair problem. The model is essentially an extension of the Peierls-Nabarro $(\mathrm{P}-\mathrm{N})$ model, in which the dislocation core is envisaged as comprising of linear elastic regions connected to one another along surfaces over which a nonlinear misfit force law acts. The misfit force law is readily established as the $\gamma$ surface, which can be calculated using atomistic means for specific materials. With the introduction of the misfit surfaces, the elastic field of the dislocation no longer carries any singularities and so the introduction of the dubious inner cutoff is not necessary. In spite of this, the model is by no means a replacement of the emerging 3D MD calculations on specific materials, but instead, will serve to provide general forms of behavior amongst different materials. In other words, it will serve as a more reliable and intuitively more correct benchmark for future MD calculations than the line-tension model.

\section{EXPERIMENTAL ACTIVATION ENERGY FOR SLIP}

Before we go on to discuss kink-pair activation, let us explore a universal relation for the activation energy for slip. Measurements of activation energy $H$ for slip in bcc metals 


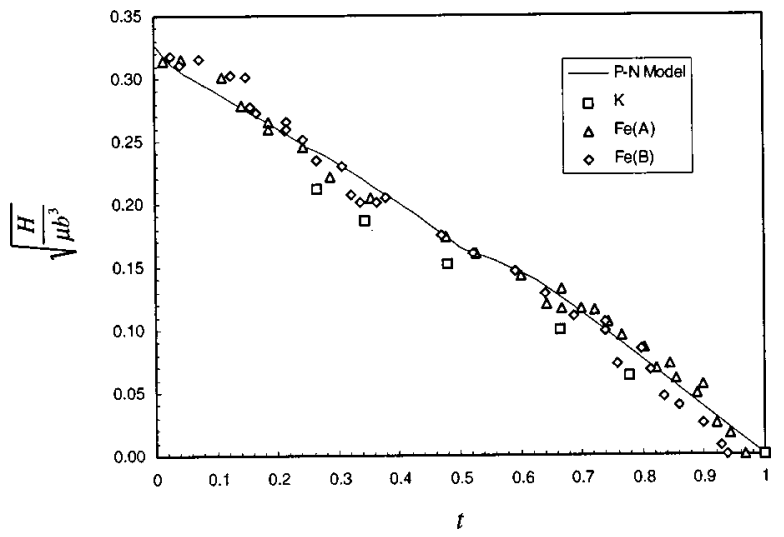

FIG. 1. Experimental activation energy vs normalized stress. $t=\tau_{a} / \tau_{p}$. Data for K from Ref. 8, Fe from Ref. 9. For Fe, data for two stress orientations are shown. Orientation $\mathrm{A}$ is close to the center of the unit triangle. $\mathrm{B}$ is close to [101].

dated back to the 60's with the pioneering work on Arrhenius analysis by Conrad and co-workers. ${ }^{7}$ These early measurements were summarized in the seminal work on kinkpair nucleation by Dorn and Rajnak; ${ }^{4}$ a feature amongst them is that they typically show large scatter and they cover only the low and intermediate stress regimes while the high stress regime is not dealt with. More reliable measurements especially those on the high stress regime were made rather recently. The results from two such reports on potassium ${ }^{8}$ and high purity iron ${ }^{9}$ are presented in Fig. 1. Here, the square root of $H$ normalized by $\mu b^{3}$, where $\mu$ is the $\langle 111\rangle$ shear modulus and $b$ the $1 / 2\langle 111\rangle$ Burgers vector, is plotted against $t$, the applied stress $\tau_{a}$ normalized by the zero-temperature Peierls stress $\tau_{p}$. It can be seen that the experimental data for both $\mathrm{K}$ and $\mathrm{Fe}$ fall on roughly the same relationship

$$
\sqrt{H / \mu b^{3}} \approx(0.30 \pm 0.03) \times(1-t)
$$

or

$$
H \approx 0.1 \mu b^{3}(1-t)^{2},
$$

where $t=\tau_{a} / \tau_{p}$. For the case of Fe, the $H$ vs $\tau_{a}$ relationship is dependent on the tensile orientation but the $H$ vs $t$ relationship is roughly orientation independent as illustrated in Fig. 1. In view of the large scatter in the early experimental data for other bcc metals, these data are not plotted in Fig. 1, but as an indication of the validity of Eq. (1), one can compare the $H$ values at zero stress from these measurements with the prediction from Eq. (1). Equation (1) predicts that $\sqrt{H(t=0) / \mu b^{3}} \approx 0.3$. Table I shows the experimental values of $\sqrt{H(t=0) / \mu b^{3}}$ from the early measurements, which indicate clearly that $\sqrt{H(t=0) / \mu b^{3}}$ falls in a narrow range 0.30 \pm 0.03 , in full agreement with Eq. (1).

The main justification for calling Eq. (1) a universal relation here is that $H(t=0)$ or $\mu b^{3}$ varies by as much as 20-folds, from $\mu b^{3}=1 \mathrm{eV}$ for $\mathrm{K}$ to $20 \mathrm{eV}$ for $\mathrm{W}$, but $\sqrt{H(t=0) / \mu b^{3}}$ varies by at most $\sim 10 \%$ about the value 0.3 (see Table I). Since $\mu b^{3}$ is the atomic unit for elastic energy, the fact that $H$ scales by $\mu b^{3}$ for a wide range of materials suggests that the activation process for slip in bcc is dominated by the elastic response of the dislocations; the influence of nonlinear bond character is only of secondary importance. While the general sharp drop of $H$ vs $t$ has been the subject of numerous investigations in the literature, to the best knowledge of the present authors, Eq. (1) has not been explicitly stated as a universal relation or its significance noticed beforehand. For this reason, the prime objective of the present article is to seek an understanding for this universal behavior from an elasticity point of view.

The activation problem of screw dislocation mobility in terms of kink-pair nucleation and expansion in the bcc structure has been investigated in the literature by the two groups of continuum theory described in Sec. I. In the the linetension theory by Dorn and Rajnak, ${ }^{4}$ a prediction is that

$$
H\left(\tau_{a}=0\right)=2 U_{k} \sim b \sqrt{\Gamma_{0} \Delta \Gamma / 2},
$$

where $U_{k}$ is the energy of an isolated kink, $\Gamma_{o}$ the line tension, and $\Delta \Gamma$ the height of the Peierls potential. ${ }^{10}$ If Eq. (1) is to be obeyed, $\sqrt{\Gamma_{o} \Delta \Gamma} /\left(\mu b^{2}\right)$ must be a constant independent of material and orientation, but there is no provision in the theory which explains why this might be so. The rather illdefined parameters such as $\Gamma_{o}$ and $\Delta \Gamma$ are obtained by fitting to experimental data for $H\left(\tau_{a}=0\right)$ and $\tau_{p}$. These fitted parameters are then used to generate flow stress versus temperature curves which are found to be in good agreement with experiment, or in the case of the investigation by Suzuki et al., ${ }^{11}$ to derive the conclusion that the Peierls potential has a flat maximum or an intermediate minimum. Thus, despite that the line-tension theory may give useful predictions, it is certainly not fundamental enough, and it cannot explain the universal relation in Eq. (1). In the "dissociation" theory by Duesbury, ${ }^{5}$ two main regimes, with the possibility of an intermediate regime in between, are identified. In the low stress regime, the long range elastic interaction of the two opposite kinks dominate, and at the stress free condition,

\begin{tabular}{|c|c|c|c|c|c|c|c|c|}
\hline & $\mathrm{Cr}$ & $\mathrm{Ta}$ & V & Mo & $\mathrm{W}$ & $\mathrm{Nb}$ & $\mathrm{K}$ & $\mathrm{Fe}$ \\
\hline$\mu b^{3}(\mathrm{eV})$ & 11.2 & 10.6 & 5.2 & 15.3 & 20.5 & 5.5 & 1.1 & 8.7 \\
\hline$\sqrt{\frac{H\left(\tau_{a}=0\right)}{\mu b^{3}}}$ & $0.25-0.30$ & $0.28-0.31$ & 0.34 & 0.30 & 0.28 & 0.30 & 0.29 & 0.32 \\
\hline References & \multicolumn{5}{|c|}{ Experimental data quoted in Dorn and Rajnak (Ref. 4) } & 7 & 8 & 9 \\
\hline
\end{tabular}

$$
H\left(\tau_{a}=0\right)=4 E_{c}+2 b^{2} \sqrt{\mu \Delta E},
$$

TABLE I. Experimental values of zero-stress activation energies.

Note: $\mu=\langle 111\rangle$ shear modulus $=\frac{2}{3} \mathrm{C}_{44}+\frac{1}{6}\left(\mathrm{C}_{11}-\mathrm{C}_{12}\right)$. 
where $E_{c}$ is the constriction energy, and $\Delta E$ the line-energy difference between the glissile and sessile states of the dislocation. Because the Volterra model was used for the fractional dislocations, both $E_{c}$ and $\Delta E$ depend on the inner cutoff of the core and the energies of the assumed stacking faults. Therefore, there is again no explicit provision that $H\left(\tau_{a}=0\right) /\left(\mu b^{3}\right)$ must be a constant. In the high stress regime, the nucleation of kink pair becomes rate controlling, and the theory predicts that

$$
H=2 E_{c}+1.3 \times \frac{\sqrt{\mu \Delta E^{3}}}{\tau_{a}} .
$$

Both $E_{c}$ and $\Delta E$ are stress dependent, but the resultant functional form of $H(t)$ does not agree with the parabolic form in Eq. (1).

\section{THE GENERALIZED PEIERLS-NABARRO MODEL}

Let us turn our attention to a new approach with a view to explain the universal stress dependence of activation in the foregoing section. The starting point here is that the screw dislocation is threefold dissociated and so the dislocated crystal is considered to be composed of three $120^{\circ}$ elastic wedges as shown in Fig. 2. The wedges are strained into an antiplane strain manner so that when they are welded together at the far field, the long-range field of the screw dislocation is established there. The wedge boundaries represent the dissociation planes where the $b / 3$ fractional dislocations lie. For bcc metals exhibiting this type of degenerate threefold core, two types of slip behavior are found. ${ }^{12,13}$ The first is the " $J_{2}$ " behavior in which spreading is wide, i.e., up to a few atomic rows, on three intersecting $\{110\}$ planes. ${ }^{12}$ The slip plane at zero temperature is always $\{110\}$ irrespective of the orientation of the maximum resolved shear stress (mrss) plane. Such slip behavior is found in metals like Fe. ${ }^{13}$ The second behavior is the $J_{1}$ behavior in which spreading is narrower so that it is more difficult to specify the dissocia-

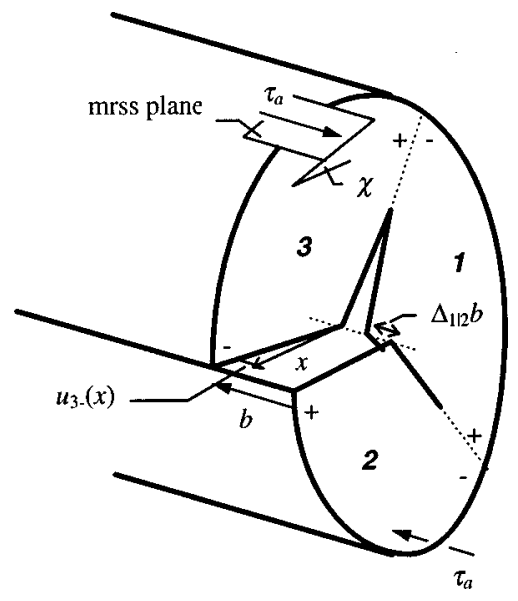

FIG. 2. The threefold core in the antiplane strain condition.

tion plane. When stressed, however, the core always moves along the twinning or antitwinning $\{112\}$ plane depending on the mrss plane, and so the dissociation plane may be considered to be $\{112\}$. This type of slip behavior is found in materials like $\beta$ brass. ${ }^{13}$ In the modeling exercise here, the wedge boundaries can therefore be taken to mean either $\{110\}$ or $\{112\}$, depending on whether the material is $J_{1}$ or $\mathrm{J}_{2}$ like.

The interaction between adjacent wedge faces in Fig. 2 is described by a nonlinear force law $\gamma[\Phi]$, where $\Phi$ is the misfit displacement. The total energy per unit length $E_{\text {tot }}$ of the dislocation is composed of three parts: (i) the strain energies of the wedges, (ii) the misfit energy of the three cuts, and (iii) the work done by the applied stress $\tau_{a}$. Each of these energy terms is a functional of the boundary displacement functions $u_{i \pm}$ defined for each wedge relative to the position of the wedge tip. $E_{\mathrm{tot}}$ can be expressed as: ${ }^{14}$

$$
\begin{aligned}
E_{\mathrm{tot}}= & \frac{3 \mu}{4 \pi} \sum_{i=1}^{3} \int_{0}^{\infty} \int_{0}^{\infty}\left\{\frac{\eta^{1 / 2}}{\xi^{3 / 2}+\eta^{3 / 2}}\left[u_{i-}^{\prime}(\xi) u_{i+}(\eta)+u_{i+}^{\prime}(\xi) u_{i-}(\eta)\right]-\frac{\eta^{1 / 2}}{\xi^{3 / 2}-\eta^{3 / 2}}\left[u_{i+}^{\prime}(\xi) u_{i+}(\eta)+u_{i-}^{\prime}(\xi) u_{i-}(\eta)\right]\right\} d \eta d \xi \\
& +\int_{0}^{\infty} \gamma\left[\Phi=\Delta_{1 \mid 2} b-u_{1-}(r)-u_{2+}(r)\right] d r+\int_{0}^{\infty} \gamma\left[\Phi=\left(1-\Delta_{1 \mid 2}-\Delta_{3 \mid 1}\right) b-u_{2-}(r)-u_{3+}(r)\right] d r \\
& +\int_{0}^{\infty} \gamma\left[\Phi=\Delta_{3 \mid 1} b-u_{3-}(r)-u_{1+}(r)\right] d r+\tau_{a} \cos \left(120^{\circ}-\chi\right) \int_{0}^{\infty}\left[\Delta_{1 \mid 2} b-u_{1-}(r)-u_{2+}(r)\right] d r+\tau_{a} \cos \chi \\
& \times \int_{0}^{\infty}\left[\left(1-\Delta_{1 \mid 2}-\Delta_{3 \mid 1}\right) b-u_{2-}(r)-u_{3+}(r)\right] d r+\tau_{a} \cos \left(120^{\circ}+\chi\right) \int_{0}^{\infty}\left[\Delta_{3 \mid 1} b-u_{3-}(r)-u_{1+}(r)\right] d r,
\end{aligned}
$$

where the double integral terms represent the strain energy, the $\gamma$ terms the misfit energy, and the $\tau_{a}$ terms the work done against the applied stress $\tau_{a}$. In this equation, $\Delta_{i \mid j}$ is the fractional Burgers vector content of the $i \mid j$ cut, and $\chi$ the angle between the mrss plane and the eventual slip plane, i.e., the $2 \mid 3$ cut in Fig. 2. At equilibrium without an applied stress, the core should adopt the symmetrical threefold configuration for which $\Delta_{1 \mid 2}=\Delta_{3 \mid 1}=1 / 3$, and when the core becomes planar along the eventual slip plane under thermal agitation or a large enough $\tau_{a}, \Delta_{1 \mid 2}=\Delta_{3 \mid 1}=0$. 
It has been shown previously ${ }^{15,16}$ that the energy per unit length of the planar state is higher than the threefold dissociated state by an amount $\Delta E \sim 0.03 \mu b^{2}$. Recombination of the core from the threefold to the planar state therefore represents an activation problem governed by the energy functional in Eq. (2). The minimum energy path for core recombination may be calculated by minimizing this energy functional under a constraint which prevents the configuration from rolling backward along the reaction coordinate. Viewing the applied stress $\tau_{a}$ as such a constraint, a point on the minimum energy path therefore represents the static configuration of the core under a given $\tau_{a}$, which is also obtained by minimizing the energy functional in Eq. (2). The minimum energy path at a fixed mrss plane orientation $\chi$ is therefore the path of static configurational change upon increasing $\tau_{a}$ at that $\chi$. Since the $\chi$ value does not matter at $\tau_{a}=0$, the minimum energy paths for different $\chi$ values should start at the same configurational point, i.e., the stressfree, threefold symmetrical configuration.

The reaction coordinate $\alpha$ along the minimum energy path is obviously an increasing function of the normalized stress $\tau_{a} / \tau_{p}$, where $\tau_{p}$ is the stress required to fully recombine the core. When the symmetrical threefold core is recombined into a partially planar configuration, the energy change referred to in Eq. (2) may be written as

$$
\Delta E_{\mathrm{tot}}=\Delta E_{\mathrm{self}}-\tau_{a} V^{*},
$$

where $\Delta E_{\text {self }}$ comprises of the strain and misfit energy contributions, and the $\tau_{a}$ term in which $V^{*}$ is the activation volume represents the work done. Within Eshelby's superposition spirit, ${ }^{17}$ this work done term is equal to the summation over the cuts $i \mid j$ of the products of the stress component acted on each cut and the area change of the cut $\delta A_{i \mid j}$ during the recombination:

$$
\begin{aligned}
\tau_{a} V^{*}= & \tau_{a} \cos \left(120^{\circ}-\chi\right) \delta A_{1 \mid 2}(\alpha, \chi)+\tau_{a} \cos \chi \\
& \times \delta A_{2 \mid 3}(\alpha, \chi)+\tau_{a} \cos \left(120^{\circ}+\chi\right) \delta A_{3 \mid 1}(\alpha, \chi),
\end{aligned}
$$

and so the activation volume

$$
\begin{aligned}
V^{*}= & \cos \left(120^{\circ}-\chi\right) \delta A_{1 \mid 2}(\alpha, \chi) \\
& +\cos \chi \delta A_{2 \mid 3}(\alpha, \chi)+\cos \left(120^{\circ}+\chi\right) \delta A_{3 \mid 1}(\alpha, \chi) .
\end{aligned}
$$

One way to define the reaction coordinate $\alpha$ is to make it proportional to $V^{*}$. Figure 3 shows the variation of $V^{*} / \cos \chi$ against $\tau_{a} / \tau_{p}$ along the equilibrium path calculated by minimizing Eq. (2) with a Frenkel force law using a RayleighRitz scheme. The results suggest that we can write

$$
V^{*}=A^{*} \alpha\left(\tau_{a} / \tau_{p}\right) \cos \chi,
$$

where $\alpha(t)$ is a function satisfying $\alpha(0)=0$ and $\alpha(1)=1$, and $A^{*}$ is a normalization constant equal to a few $b^{2}$. Along the minimum energy path, the stress-free, threefold state is represented by $\alpha=0$, and the fully recombined activated point by $\alpha=1$.

Let us turn to the energy potential along the minimum energy path. Figure 4 shows the stress-induced self-energy change $\Delta E_{\text {self }}$ obtained by minimizing Eq. (2). The results

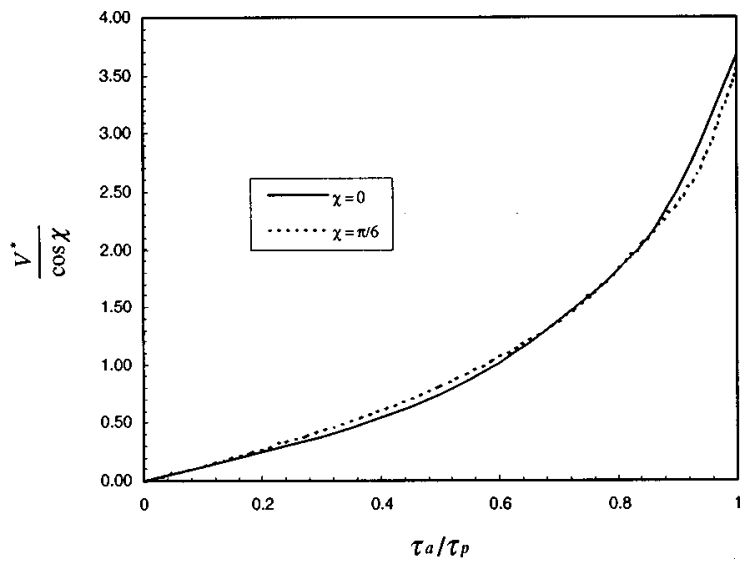

FIG. 3. Activation volume $V^{*}$ for core recombination as a function of normalized applied stress for different stress orientation $\chi$.

are plotted against the reaction coordinate $\alpha$ defined above. It can be seen that the $\Delta E_{\text {self }}(\alpha)$ relation can be represented by a phenomenological parabolic relationship:

$$
\Delta E_{\text {self }}(\alpha) \approx\left\{\begin{array}{l}
\frac{\Delta E^{2}}{c \cos \chi}, \quad \text { for } 0 \leqslant \alpha \leqslant c \\
\frac{\Delta E}{\cos \chi}\left[1-\frac{(\alpha-1)^{2}}{1-c}\right], \quad \text { for } c \leqslant \alpha \leqslant 1,
\end{array}\right.
$$

where $\Delta E$ is the activation barrier at $\chi=0^{\circ}$ and $c$ is a constant between 0 and 1 used to characterize the shape of the energy potential (see Fig. 5). With Eqs. (3b) and (4), $\Delta E_{\text {tot }}$ in Eq. (2b) can be minimized with respect to $\alpha$ to yield a relation between the static degree of recombination $\alpha_{s}$ under a given applied stress. The zero-temperature Peierls stress $\tau_{p}$ is the value of $\tau_{a}$ when $\alpha_{s}=c$, and is given by

$$
\tau_{p}=\frac{2 \Delta E}{A^{*} \cos ^{2} \chi} \text {. }
$$

\section{KINK-PAIR NUCLEATION}

At finite temperatures, the dislocation will not move as a rigid line. In the high stress or low temperature regime, the

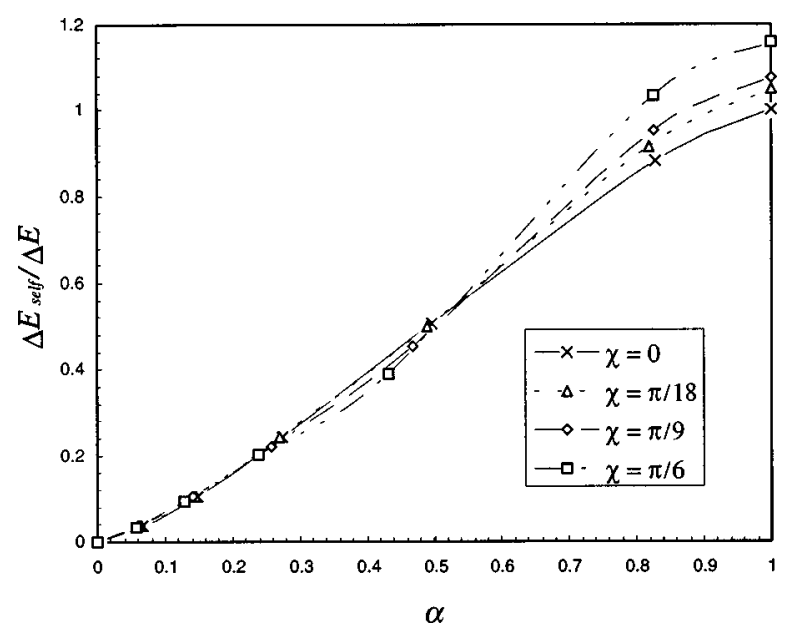

FIG. 4. $\Delta E_{\text {self }}(\alpha)$ along minimum energy path for different orientation $\chi$. 


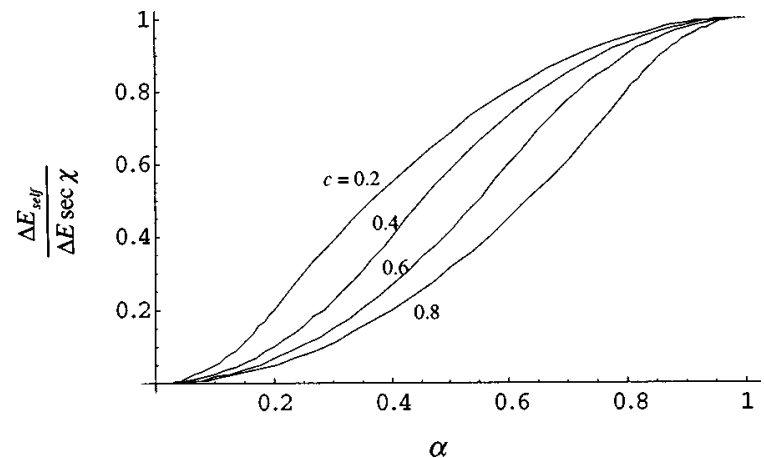

FIG. 5. Schematic minimum energy path of the recombination process for different values of $c$

activation process involves a saddle configuration in the form of a kink-pair illustrated schematically in Fig. 6. In this configuration, the dislocation core changes continuously from the fully dissociated state into the fully recombined state through a kink, and then back to the fully dissociated state through another kink with opposite sign as shown in Fig. 6 . The geometry represents a 3D elasticity problem and the mathematics can be greatly simplified by invoking a slab approximation, in which we assume that for each slab perpendicular to the dislocation line, the local degree of recombination can be characterized by the reaction coordinate $\alpha$ defined above, whose value varies from $\alpha_{s} \approx 0$ for the fully dissociated state to $\alpha_{0} \approx 1$ for the fully recombined state. The kink shape is then marked by the function $\alpha(\eta)$, where $\eta$ is the spatial coordinate along the dislocation line.

Let us denote in polar coordinates the screw direction displacement $u(r, \theta)$ of the configuration at point $\alpha$ on the minimum energy path at $\chi$ by

$$
u(r, \theta)=\Delta u^{\chi}(r, \theta, \alpha)+u^{\Delta}(r, \theta),
$$

where $u^{\Delta}(r, \theta)$ is the displacement of the threefold reference state at $\alpha=0$. For a varying configuration $\alpha(\eta)$ under the slab approximation, the normal strain $\epsilon_{\eta \eta}$ in the dislocation direction $\eta$ is

$$
\epsilon_{\eta \eta}=\frac{\partial u}{\partial \eta}=\alpha^{\prime}(\eta) \frac{\partial \Delta u^{\chi}(r, \theta, \alpha)}{\partial \alpha} .
$$

The interaction energy between slabs for a given $\alpha(\eta)$ is:

$$
E_{\mathrm{int}}=\frac{E}{2} \int d \eta \iint \epsilon_{\eta \eta^{2}}^{2} r d r d \theta=\frac{E}{2} \int\left[\alpha^{\prime}(\eta)\right]^{2} P d \eta,
$$

where $E$ is an effective Young's modulus local to the dislocation core region, and

$$
P=\iint\left[\frac{\partial \Delta u^{\chi}(r, \theta, \alpha)}{\partial \alpha}\right]^{2} r d r d \theta .
$$

With the definition of $\alpha$ expressed in Eq. (3b), the boundary displacements of the wedges in Fig. 2 are approximately linear with respect to $\alpha$. Since the displacement of the wedge interior is linear with respect to the boundary displacement functions, $\Delta u^{\chi}$ is approximately linear with respect to $\alpha$, and
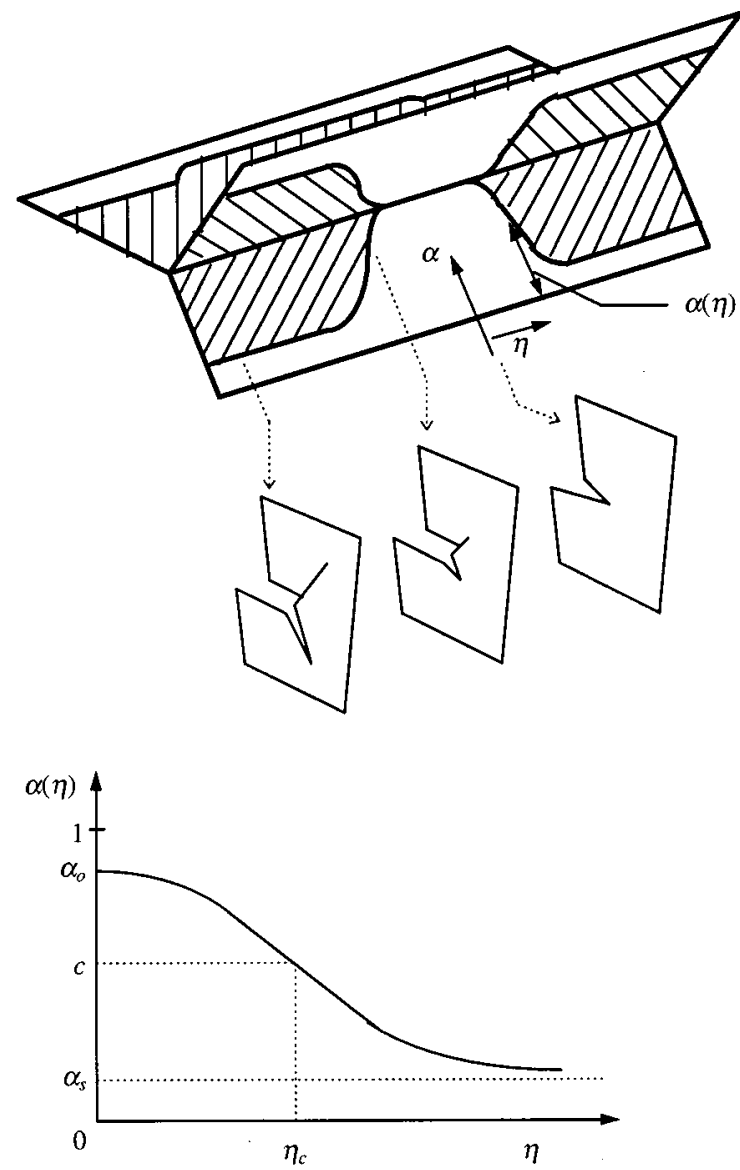

FIG. 6. Configuration of a kink pair during nucleation.

so $P$ in Eq. (6b) is approximately constant with respect to $\alpha$. Also, from Eq. (3b), $\Delta u^{\chi}$ is roughly proportional to $\cos \chi$, so that one can write

$$
P \approx P_{0} \cos ^{2} \chi,
$$

where $P_{o}$ is the value of $P$ at $\chi=0^{\circ}$. Physically, $\sqrt{P_{o}}$ is simply the root-mean-squared change in the displacement on going from the threefold to the planar configuration multiplied by an effective size of the core, and so $\sqrt{P_{o}}$ is of the order of a fraction of $b^{2}$.

From Eqs. (3b), (4), and (6), the total energy $H_{h s}$ of the whole kink pair in the high stress regime is

$$
\begin{aligned}
H_{h s}= & 2 \times \int_{0}^{\infty}\left\{\left[\Delta E_{\text {self }}(\alpha)-\Delta E_{\text {self }}\left(\alpha_{s}\right)\right]\right. \\
& \left.-\tau_{a} A^{*} \cos \chi\left(\alpha-\alpha_{s}\right)+E P \alpha^{\prime 2} / 2\right\} d \eta,
\end{aligned}
$$

where $\alpha_{s}$ marks the static antiplane strain configuration under $\tau_{a}$ and is given by

$$
\alpha_{s}=\frac{c \tau_{a} A^{*} \cos ^{2} \chi}{2 \Delta E} .
$$

The critical kink shape can be obtained by extremizing $H_{h s}$ in Eq. (7) analytically with respect to $\alpha(\eta)$. Figure 7 shows the critical kink shape for different applied stress levels specified by $t=\tau_{a} / \tau_{p}$. The critical kink height is found to decrease as the applied stress $t$ increases, and this is in quali- 


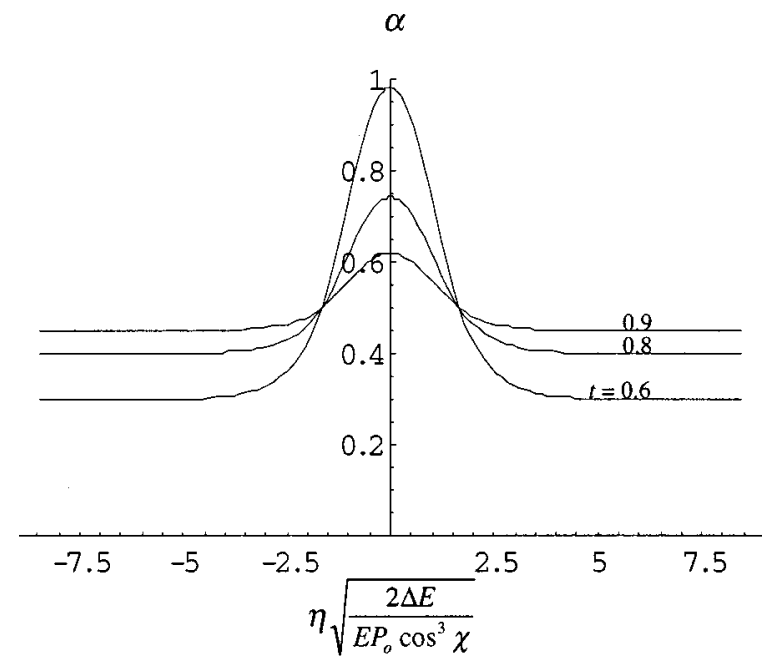

FIG. 7. Saddle point kink shape at different stress levels in the high stress regime.

tative agreement with atomistic calculations. ${ }^{18}$ In the high stress regime, $\alpha(\eta=0) \leqslant 1$. This leads to the following criterion for the high stress regime:

$$
t \geqslant \frac{1}{1+\sqrt{1-c}} \approx 0.6, \text { if } c=0.5 \text {. }
$$

The minimized value of $H_{h s}$ from Eq. (7) is found to be

$$
\begin{aligned}
H_{h s}= & (1-t)^{2} \sqrt{2(\Delta E) E P_{o} \cos \chi} \\
& \times\left[\sqrt{c}+\frac{1}{\sqrt{1-c}}\left(\pi-\tan ^{-1} \sqrt{\frac{c}{1-c}}\right)\right] .
\end{aligned}
$$

\section{KINK-PAIR EXPANSION}

In the low stress regime, the saddle configuration involves the expansion of the well-formed pair of kinks along the screw direction as depicted in Fig. 8. Each kink is connected to the screw segments on either side by two constrictions as shown. In the early treatment by Duesbury, ${ }^{5}$ the constriction energy is calculated using the Volterra field and involves ill-defined parameters such as inner cutoff, critical separation for recombination and stacking fault energies. Here we treat the constriction problem using the $\mathrm{P}-\mathrm{N}$ model taking stress into account. Using the slab approximation above, the $\alpha$ variation of a constriction is shown in Fig. 8. The essential difference between a constriction shown in Fig. 8 and a kink-pair nucleus shown in Fig. 6 is that for the kink pair, $\alpha(\eta=0) \leqslant 1$ and $\alpha^{\prime}(0)=0$ by symmetry, but for the constriction, $\alpha(0)=1$ as the configuration constricts into the planar edge segment of the kink. The constriction energy $E_{c}$ has a functional form similar to Eq. (7):

$$
\begin{aligned}
E_{c}= & \int_{0}^{\infty}\left\{\left[\Delta E_{\text {self }}(\alpha)-\Delta E_{\text {self }}\left(\alpha_{s}\right)\right]\right. \\
& \left.-\tau_{a} A^{*} \cos \chi\left(\alpha-\alpha_{s}\right)+E P \alpha^{\prime 2} / 2\right\} d \eta,
\end{aligned}
$$

but now minimization is subject to $\alpha(0)=1$ and $\alpha(\infty)$ $=\alpha_{s}$. The saddle configuration of the constriction is shown in Fig. 9 for different stress levels. The saddle point constric-
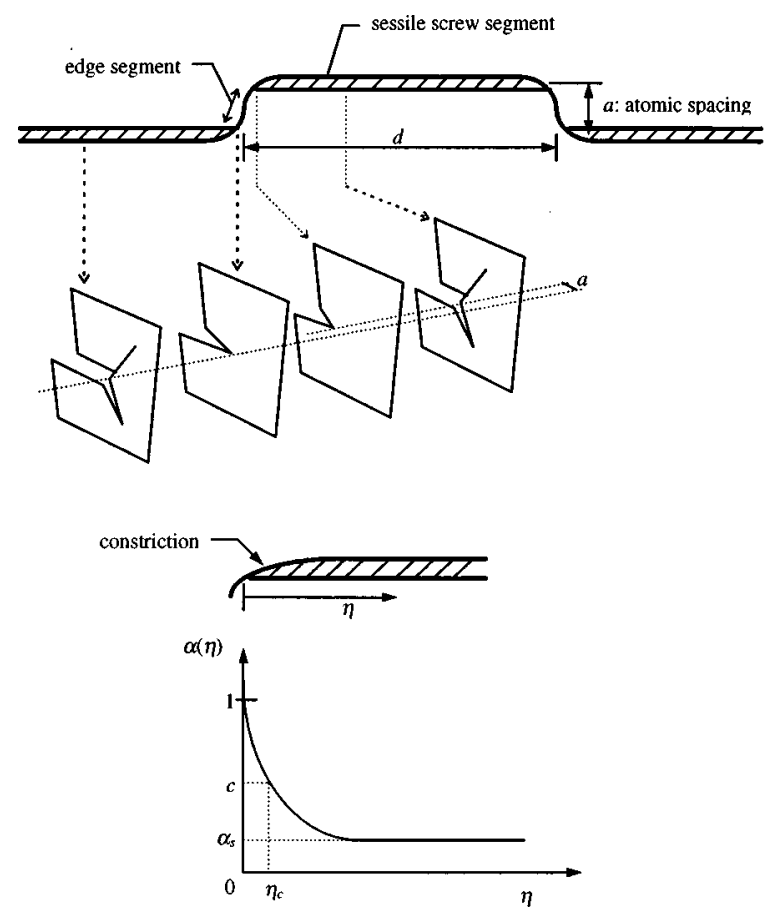

FIG. 8. Kink-pair expansion.

tion energy $E_{c}$ can be shown to be given by:

$$
E_{c}=\sqrt{\frac{(\Delta E) E P_{o} \cos \chi}{2}} g(c, t)
$$

where

$$
\begin{aligned}
& g(c, t)= \sqrt{c}(1-t)^{2}+t(1-c) \sqrt{1-2 t+c t^{2}}+(1-t)^{2} \sqrt{1-c} \\
& \times\left[\sin ^{-1}\left(\frac{t \sqrt{1-c}}{1-t}\right)+\tan ^{-1} \sqrt{\frac{1-c}{c}}\right], \\
& t \leqslant \frac{1}{1+\sqrt{1-c}} .
\end{aligned}
$$

The dependence of $E_{c}$ on $c$ and $t$ is shown in Fig. 10.

The energy $H_{l s}$ of the whole kink pair in the low stress regime comprises $4 E_{c}$ for the four constrictions, the selfenergy $2 E_{e}$ of the atomic step edge segments in the two

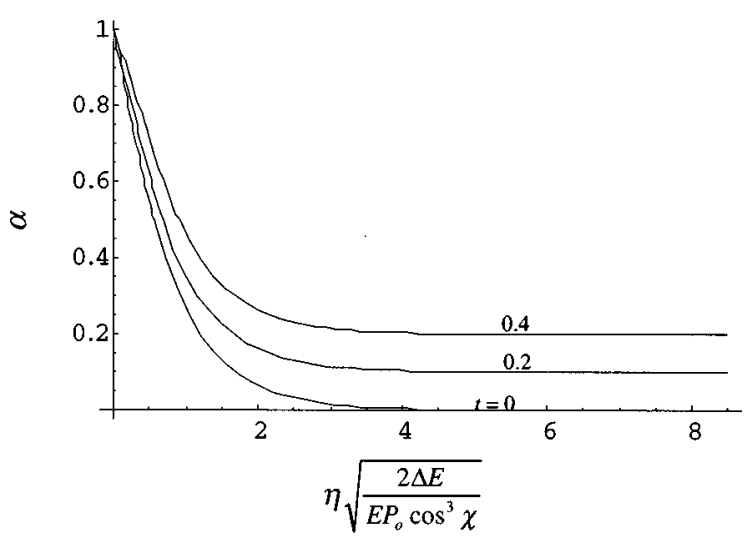

FIG. 9. Saddle point constriction shape at different stress levels in the low stress regime. 


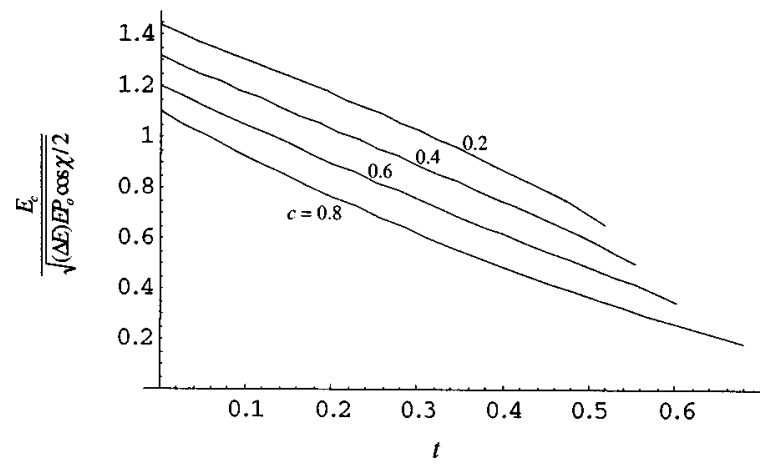

FIG. 10. Dependence of $E_{c}$ on $c$ and $t$.

kinks, the long-range interaction energy between the kinks, and the work done by stress. ${ }^{5}$ The self-energy of an elementary edge segment is roughly $a \Delta E$, where $a$ is the periodic spacing along the slip plane. For large kink separations, the interaction energy can be well modeled by using Volterra fields for the edge segments. At kink separation $d, H_{l s}$ is given by:

$$
H_{l s}=4 E_{c}+2 E_{e}-\frac{f \mu b^{2} a^{2}}{d}-\tau_{a} b a d \cos \chi,
$$

where $f=1-2 \mathrm{v} / 8 \pi(1-\mathrm{v})=0.0227$ for Poisson's ratio $\mathbf{v}$ $=0.3$. To obtain the saddle configuration, $H_{l s}$ is maximized with respect to $d$, the result is the following activation energy for the low stress regime:

$$
H_{l s}=4 E_{c}(t)+2 E_{e}-2 \mu b^{3} \sqrt{\frac{2 f \Delta E}{\mu A^{*} \cos \chi}} \sqrt{t},
$$

where we have put $a=b$ and $E_{c}(t)$ is given by Eq. (11).

\section{DISCUSSION}

\section{A. Orientation dependence of Peierls stress}

In Eq. (5), $A^{*}$ marks the area change of the cuts in Fig. 2 during recombination and is of the order of a few $b^{2}$. Taking $\Delta E$ to be $\sim 10^{-2} \mu b^{2}$ as estimated previously, ${ }^{16} \tau_{p}$ is about $10^{-2} \mu$, which agrees well with typical atomistic simulation results in the literature. ${ }^{1}$ From Eq. (5), $\tau_{p}$ is orientation dependent, showing a minimum at $\chi=0^{\circ}$. The $\sec ^{2} \chi$ variation stems from the approximations involved in Eqs. (3b) and (4), but numerical minimization of the full energy functional in Eq. (2) using a Rayleigh-Ritz method confirms the minimum at $\chi=0^{\circ}$ (see Fig. 11).

For materials with $J_{2}$-like potentials like Fe and Mo, dissociation is wide on three intersecting $\{101\}$ planes and the eventual slip plane is one of these $\{101\}$ planes irrespective of the stress orientation $\chi$. Using the conventional definition for the stress orientation in which $\chi$ is measured from a $\{101\}$ plane, the corresponding $\tau_{p^{-}} \chi$ relationship of this type of materials shows a minimum at $\chi=0^{\circ}$ and maximum at $\chi= \pm 30^{\circ}$, in qualitative agreement with the present prediction [see Fig. 12(a)]. For materials with limited dissociation on $\{101\}$ planes such as the $J_{1}$-like materials, there is a general tendency for the core to glide on a twinning or antitwinning $\{112\}$ plane depending on stress orientation. The

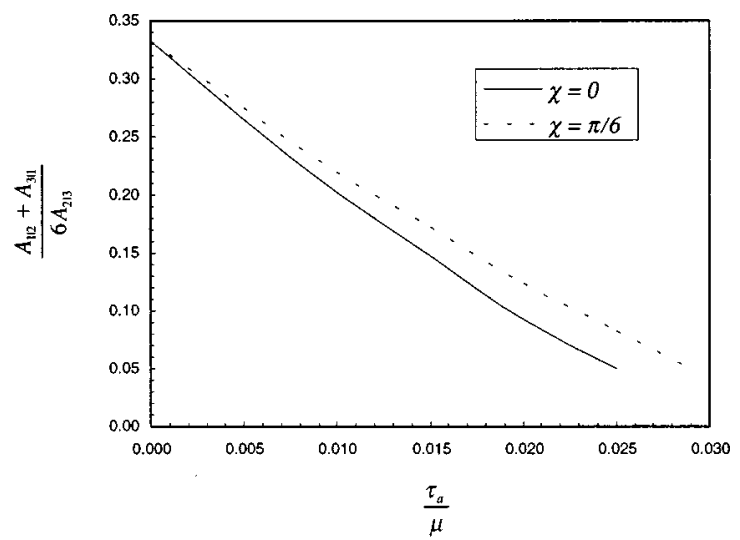

FIG. 11. Core recombination under antiplane strain condition. $A_{i \mid j}$ is the area of the $i \mid j$ cut in Fig. 2. When $\tau_{a}=0$, the three cuts have equal areas and so the $y$ intercept is $1 / 3$. As $\tau_{a}$ increases, the core recombines towards the planar configuration so that $A_{1 \mid 2}$ and $A_{3 \mid 1}$ diminish while $A_{2 \mid 3}$ enlarges.

associated $\tau_{p^{-}} \chi$ relationship would exhibit a maximum at the $\chi$ value at which the slip plane changes from the twinning to antitwinning $\{112\}$, and local minimum points at $\chi= \pm 30^{\circ}$. Because of the twinning/antitwinning asymmetry of the atomic arrangement on the $\{112\}$ plane, $\tau_{p}$ for twinning slip is in general lower than that for antitwinning slip [see Fig. 12(c)]. This type of slip behavior is found in bcc materials like $\mathrm{Li}-\mathrm{Mg}, \mathrm{AgMg}$, and $\beta-\mathrm{CuZn}$. In the present treatment, the detailed atomic arrangement along the screw direction is not modeled, and so the twinning/antitwinning asymmetry of $\tau_{p}$ is not predicted. However, by treating the three dissociation planes in Fig. 2 to be $\{112\}$ so that stress orientation angle should now be measured from a $\{112\}$ eventual slip plane, $\tau_{p}$ should still be given by Eq. (5) after replacing $\chi$ by $\left(\chi \pm 30^{\circ}\right)$, i.e.,

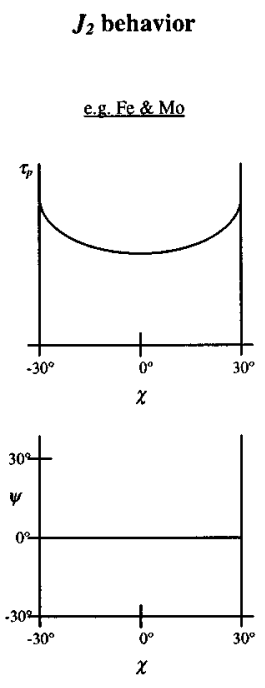

(a)
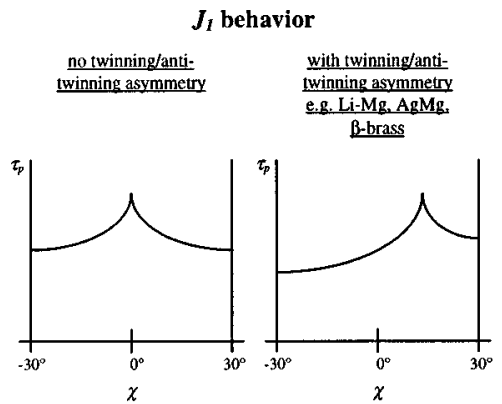

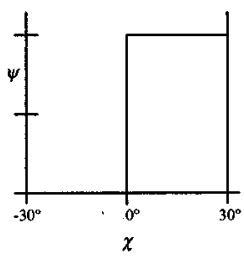

(b)

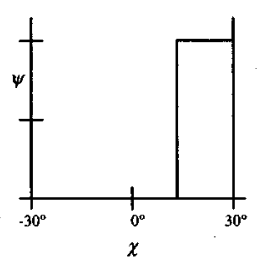

(c)

FIG. 12. Schematic $\tau_{p^{-}} \chi$ relations for $J_{2}$ and $J_{1}$ behavior. $\chi$ is the orientation of the mrss plane, measured from a $\{101\}$ reference plane towards the direction of the antitwinning $\{112\}$ plane. $\psi$ is the orientation of the actual slip plane. 


$$
\tau_{p}= \begin{cases}\frac{2 \Delta E}{A^{*} \cos ^{2}\left(\chi+30^{\circ}\right)} & -30^{\circ} \leqslant \chi \leqslant 0^{\circ}, \\ \frac{2 \Delta E}{A^{*} \cos ^{2}\left(\chi-30^{\circ}\right)} & 0^{\circ} \leqslant \chi \leqslant 30^{\circ} .\end{cases}
$$

Such a $\tau_{p}-\chi$ relationship is shown schematically in Fig. 12(b). Compared with the real behavior in Fig. 12(c), the overall form except the twinning/antitwinning asymmetry is predicted.

A third type of core configuration, namely the sixfold configuration, is found in the group VB metals $\mathrm{V}, \mathrm{Nb}$, and Ta. ${ }^{19}$ The present treatment assumes the ground state configuration to be the threefold core, and so the analysis here will not apply to the group VB metals. ${ }^{20}$ An interesting observation, however, is that the experimental $\tau_{p^{-}} \chi$ relationship for the group VB member Ta is neither the Fe type nor the $\beta$-CuZn type shown in Fig. 12. Instead the slip plane changes from the twinning $\{112\}$ to $\{101\}$ when $\chi$ approaches $30^{\circ}$.

\section{B. Kink-pair activation}

The present $\mathrm{P}-\mathrm{N}$ model predicts that in the high stress regime defined according to Eq. (8), the activation energy varies with stress according to

$$
H=C \mu b^{3}(1-t)^{2}
$$

as indicated in Eq. (9), where $C$ is perceived here as a factor roughly independent of material. Within the allowable range of $\chi$ from $0^{\circ}$ to $\pm 30^{\circ}$, the variation of $C$ as inferred from Eq. (9) is less than $7 \%$, and so the orientation dependence of $C$ is almost indiscernible. The mathematical form of Eq. (14) is identical to the experimental findings in Fig. 1 and Eq. (1); the agreement is a strong verification for the present model. Detailed analysis of Eq. (9) shows that the factor $C$ in Eq. (14) is rather insensitive to the choice of $c$ from $c=0$ to 0.8 , but it strongly depends on the value of the lumped parameter $(\Delta E) E P_{0}$. This has the important implication that knowledge of the exact shape of the minimum energy path is unimportant; the only parameter of the minimum energy path which can significantly influence $H$ is the energy barrier $\Delta E$ in Fig. 5. This is strong justification for the using a phenomenological recombination potential in the calculation. From the previous analysis by Ngan, ${ }^{16}$ the energy difference between a stable threefold core and a planar core is $\sim 0.03 \mu b^{2}$. We expect $\Delta E$ to be smaller than this because motion may start before the full planar state is reached. A reasonable value for $\Delta E$ is $0.02 \mu b^{2}$. Returning to the variation between $H$ and $t, C$ from Fig. 1 is $\sim 0.1$. In Eq. (14), $C$ will assume this value for all $c \lesssim 0.8$ when $\Delta E \sim 0.02 \mu b^{2}$ and $E P_{o} \sim 0.03 \mu b^{4}$. Recall that $\sqrt{P_{o}}$ is a core size multiplied by the root-mean-squared difference in displacement between the threefold and planar configuration, we expect $\sqrt{P_{o}}$ to be a fraction of $b^{2}$. Hence, $P_{o}$ is $\sim 0.1 b^{4}$ and so $E$ is a fraction of $\mu$. In other words, the requirement here is for the effective modulus along the dislocation core to be softer than the bulk by a few times, and this is not unreasonable.

In the low stress regime for which $t \leqslant 1 /(1+\sqrt{1-c})$, $H(t)$ in Eq. (13) appears to be more complicated than the parabolic relation in Eq. (14). However, substituting the previously concluded values of the parameters into Eq. (13) shows that the $H_{l s}(t)$ relationship is almost parabolic. For example, the choice $\Delta E=0.02 \mu b^{2}, E P_{o}=0.03 \mu b^{4}, c$ $=0.5, A^{*}=2 b^{2}, f=0.023$, and $E_{e}=0.01 \mu b^{3}$ yields a $H_{l s}(t)$ relationship from Eq. (13) almost continuous to the $H_{h s}(t)$ relationship from Eq. (9). The whole $H(t)$ relationship obtained as the union of the low and high stress branches is shown as the thin solid line in Fig. 1. The agreement with experimental results is excellent.

Here, the claim to have satisfactorily explained the universal relation in Eq. (1) is made based upon the belief that the variations of the normalized parameters $\Delta E /\left(\mu b^{2}\right)$ and $E P_{o} /\left(\mu b^{4}\right)$ are small amongst materials. The conclusion that $\Delta E \sim 0.02-0.03 \mu b^{2}$ was made previously by directly solving the generalized Peierls-Nabarro integral equations using a generic Frenkel force law. ${ }^{16}$ Small deviations of the $\gamma$ surface from the sinusoidal form are expected to result in only fractional changes in $\Delta E$, which will not upset significantly the constancy of $C$ in Eq. (14). $E P_{o} \sim 0.03 \mu b^{4}$ is based on the physical interpretation of the parameter, and again, reasonable changes in the factor 0.03 will not result in significant changes in the value of $C$. As an indication, since the vertical intercept in Fig. 1 is proportional to $\sqrt[4]{(\Delta E) E P_{o}}$, doubling the lumped parameter $(\Delta E) E P_{o} /\left(\mu^{2} b^{6}\right)$ will result in only $19 \%$ increase in the intercept.

It is interesting to note that, in the high-stress regime, the line-tension treatment of Dorn and Rajnak ${ }^{4}$ would also yield a parabolic relation between $H$ and $t$ similar to Eq. (14) if a piecewise parabolic relation is used for the Peierls potential. This is because the kink energy functional Eq. (7) has a mathematical form similar to that in the line-tension treatment, with $\Delta E_{\text {self }}$ analogous to the Peierls potential, $E P / b^{2}$ analogous to the line-tension $\Gamma_{o}$, and $A^{*}$ analogous to the quantity $b^{2}$ in the line-tension treatment. The physical limitations of the line-tension approach have already been discussed in the Introduction. The present treatment replaces the phenomenological Peierls potential concept by the recombination potential concept as manifested in Eqs. (2) and (4). The recombination potential of Eq. (4) and Fig. 5 is defined with respect to the reaction coordinate $\alpha$ along the minimum energy path, which, unlike the Peierls potential, is well prescribed according to Eq. (3b) once the minimum energy path is located. The quantity EP measures the rigidity of the core towards bow out, and, like its counterpart $\Gamma_{o}$ in the linetension approach, is only known by its order of magnitude. However, the EP concept is intuitively more accurate than the line-tension concept. Finally, in the line-tension treatment, the work done is the force on unit length of the dislocation $\tau_{a} b$ multiplied by the area "swept" by the dislocation line. In the present model, the work done is calculated according to Eq. (3) by considering the core configuration change during the recombination process. The present treatment is clearly much more physical.

\section{CONCLUSIONS}

The movement of $1 / 2\langle 111\rangle$ screw dislocations in bcc metals exhibiting the threefold core structure can be modeled 
within the framework of the generalized Peierls-Nabarro model. The apparent activation energy is predicted to vary with stress in an approximately parabolic manner. This is in good agreement with experimental findings for different bcc materials.

\section{ACKNOWLEDGMENT}

This research was supported by an earmarked research grant (No. HKU 572/96E) from the Research Grants Council, Hong Kong.

${ }^{1}$ M. S. Duesbury, in Dislocations in Solids, Vol. 8-Basic Problems and Applications, edited by F. R. N. Nabarro (North-Holland, Amsterdam, 1989), pp. 67-173.

${ }^{2}$ Materials Research Society Symposium Proceedings, Vol. 538, edited by V. Bulatov, N. Ghoniem, T. D. de la Rubia, and T. Kaxiras (to be published).

${ }^{3}$ V. Celli, M. Kabler, T. Ninomiya, and R. Thomson, Phys. Rev. 131, 58 (1963).

${ }^{4}$ J. E. Dorn and S. Rajnak, Trans. AIME 230, 1052 (1964).

${ }^{5}$ M. S. Duesbury, Philos. Mag. 19, 501 (1969).

${ }^{6}$ V. Vitek, Philos. Mag. 18, 773 (1968).

${ }^{7}$ H. Conrad and W. Hayes, Trans. ASM 56, 249 (1963).
${ }^{8}$ Z. S. Basinski, M. S. Duesbury, and G. S. Murty, Acta Metall. 29, 801 (1981).

${ }^{9}$ Y. Aono, E. Kuramoto, and K. Kitajima, Rep. Res. Inst. Appl. Mechanics, Kyushu Univ. 29, 127 (1981); reprinted in Ref. 13, pp. 94/95.

${ }^{10}$ P. Guyot and J. E. Dorn, Can. J. Phys. 45, 983 (1967).

${ }^{11}$ T. Suzuki, H. Koizumi, and H. O. K. Kirchner, Acta Metall. Mater. 43, 2177 (1995).

${ }^{12}$ V. Vitek, Cryst. Lattice Defects 5, 1 (1974).

${ }^{13}$ T. Suzuki, S. Takeuchi, and H. Yoshinga, Dislocation Dynamics and Plasticity (Springer, Berlin, 1991), Chap. 6.

${ }^{14}$ A. H. W. Ngan, Physica A (to be published).

${ }^{15}$ A. H. W. Ngan, Philos. Mag. Suppl. 72, 207 (1995).

${ }^{16}$ A. H. W. Ngan, J. Mech. Phys. Solids 45, 903 (1997).

${ }^{17}$ J. D. Eshelby, Philos. Trans. R. Soc. London, Ser. A 244, 87 (1951).

${ }^{18}$ M. S. Duesbury, Acta Metall. 31, 1747 (1983).

${ }^{19}$ M. S. Duesbury and V. Vitek, Acta Mater. 46, 1481 (1998).

${ }^{20}$ The $\gamma$ surfaces for VB metals are found to be sinusoidal-like with a small plateau at the top. The existence of the sixfold core in these metals is highly plausible from the Peierls-Nabarro model point of view, as the analysis by Ngan (Refs. 15 and 16) suggests that higher fold symmetry is more favorable because it helps spread the strain more effectively from the dislocation center. For group VIB metals $(\mathrm{Cr}, \mathrm{Mo}, \mathrm{W})$, Duesbury and Vitek (Ref. 19) found that the $\gamma$ surface has a shoulder at the $b / 3$ position, and this is a factor favoring the stabilization of the threefold core. This may well be the reason for the exhibition of the threefold core in other Fe-like materials. Detailed P-N analysis of the effect of the shape of $\gamma$ surface on the ground state core configuration is underway. 